\title{
Current and emerging treatments for the management of myasthenia gravis
}

This article was published in the following Dove Press journal:

Therapeutics and Clinical Risk Management

$2 \mathrm{I}$ July $20 \mathrm{II}$

Number of times this article has been viewed

\author{
Sivakumar Sathasivam \\ The Walton Centre NHS Foundation \\ Trust, Lower Lane, Liverpool, UK
}

\begin{abstract}
Myasthenia gravis is an autoimmune neuromuscular disorder. There are several treatment options, including symptomatic treatment (acetylcholinesterase inhibitors), short-term immunosuppression (corticosteroids), long-term immunosuppression (azathioprine, cyclosporine, cyclophosphamide, methotrexate, mycophenolate mofetil, rituximab, tacrolimus), rapid acting short-term immunomodulation (intravenous immunoglobulin, plasma exchange), and long-term immunomodulation (thymectomy). This review explores in detail these different treatment options. Potential future treatments are also discussed.
\end{abstract}

Keywords: acetylcholinesterase inhibition, immunosuppression, immunomodulation, thymectomy

\section{Introduction}

Myasthenia gravis (MG) is a neuromuscular disorder characterized by a clinical course of fluctuating, painless muscle weakness. Typically it begins in the extraocular muscles and remains purely ocular in $15 \%$ of patients. MG generalizes by descending to involve the bulbar muscles, and subsequently the neck, proximal limb, and sometimes respiratory muscles. Even with modern treatments, at least $20 \%$ of patients experience a myasthenic crisis which requires intubation and mechanical ventilation, usually within the first 2 years of the diagnosis.

MG is an autoimmune disorder usually caused by antibodies to postsynaptic proteins, mainly nicotinic acetylcholine receptor (AChR) and muscle-specific kinase (MuSK), but there are other as yet undiscovered antigens. These antibodies reduce the number of functional AChRs and thus impair neuromuscular transmission. The prevalence of MG has increased from around 5 per million population between 1915 and $1934^{1}$ to about 200 per million population now, ${ }^{2}$ in part due to improved detection of the antibodies to the postsynaptic proteins. The estimated annual incidence of MG is between 1 in 10,000 to 1 in 50,000 of the population, ${ }^{3}$ but the clinical recognition of this rare disease remains difficult with many patients going undiagnosed for many months from symptom onset, and the diagnosis only correctly made after several physician consultations. In the past 70 years, treatment advances have reduced the mortality of MG from 70\% between 1915 and $1934^{1}$ to $5 \%$ or less now. ${ }^{4}$

In this review an overview will be given of the mechanism, evidence, indication, and relevant adverse effect profile of the different treatment options in generalized MG. Several potential future therapies will also be discussed.
Correspondence: Sivakumar Sathasivam The Walton Centre NHS Foundation Trust, Lower Lane, Liverpool L9 7LJ, UK Tel +44 I5I5298I5I

$\mathrm{Fax}+44$ I5I52955I2

Email sivakumar.sathasivam@

thewaltoncentre.nhs.uk 


\section{Symptomatic treatment Acetylcholinesterase inhibitors}

In MG, the first-line option is symptomatic treatment with acetylcholinesterase inhibitors. Pyridostigmine bromide is the most commonly used drug. Other acetylcholinesterase inhibitors such as neostigmine are rarely used because of their poorer pharmacodynamic profiles and tolerability. In an observational study of 14 MG patients comparing pyridostigmine with neostigmine, it was concluded that over 1 year, pyridostigmine was more effective with less adverse events. ${ }^{5}$ Similar conclusions were reached in another observational study of 69 patients which compared the use of pyridostigmine with neostigmine. ${ }^{6}$ There is no large randomized controlled trial of acetylcholinesterase inhibitors in $\mathrm{MG}$, but the clear response of this drug in observational studies would make depriving patients in the placebo arm of a randomized controlled trial unethical and unjustifiable.

Pyridostigmine is most effective early in the course of MG and over time increasing tolerance to the drug develops which may necessitate dose escalation. Most MG patients do not achieve adequate response with acetylcholinesterase inhibitor treatment and will require further immunosuppression. It is also noteworthy that some MuSK antibody-positive patients may show nonresponsiveness to acetylcholinesterase inhibitors. In one study, $71 \%$ of MuSK antibody positive patients failed to respond to acetylcholinesterase inhibitors, compared to $18 \%$ respectively of $\mathrm{AChR}$ antibody positive and seronegative patients. ${ }^{8}$ Pyridostigmine is generally well tolerated. Adverse events include muscarinic side effects such as nausea, vomiting, abdominal cramping, diarrhea, diaphoresis, increased lacrimation, excessive respiratory secretions, bradycardia, and atrioventricular block. Antimuscarinics such as propantheline bromide provide effective symptomatic relief against the abdominal adverse events induced by pyridostigmine. Pyridostigmine may also cause nicotinic adverse events such as muscle cramps and fasciculations, but these rarely require a change in the dose of the drug. High doses of pyridostigmine may desensitize AChRs and induce weakness resulting in a cholinergic crisis. If there is such a concern, cholinesterase inhibitors need to be temporarily withdrawn and the patient carefully monitored for improvement.

\section{Short-term immunosuppression Corticosteroids}

Corticosteroids are thought to act on the immune system by inhibiting the activation of T-cells and impairing the function of cells of the monocyte/macrophage lineage. Adrenocorticotrophic hormone (ACTH) was first described to have a beneficial effect in MG in 1935. ' 'Good improvement' was reported in a study of 100 patients with severe refractory MG given ACTH. ${ }^{10}$ In four large retrospective studies of generalized MG using various doses of corticosteroids and with different follow-up durations, $74 \%$ of a total of 422 patients achieved good overall improvement of muscle strength or remission. ${ }^{11-14}$ A prospective study of $600 \mathrm{MG}$ patients (151 generalized, 449 pure ocular) treated with moderate doses of corticosteroids followed by low-dose maintenance showed an overall improvement in $95 \%$ of cases, but no clear breakdown between the generalized and ocular cases were given. ${ }^{15}$

A randomized double-blind trial of prednisolone versus placebo in 13 patients with generalized MG showed no significant improvement of muscle strength at 6 months. ${ }^{16}$ Another randomized double-blind trial of intravenous methylprednisolone versus placebo in 19 patients with generalized MG showed a significant short-term benefit from corticosteroids 2 weeks after treatment. ${ }^{17}$ An openlabel randomized trial comparing high-dose intravenous methylprednisolone and low-dose oral prednisolone in 39 patients with juvenile MG (eight generalized and 31 ocular) did not report any significant difference in improvement between the two groups, although the exact time of measurement and breakdown between the generalized and ocular cases were unclear from the paper. ${ }^{18}$

Corticosteroids are useful as short-term immunosuppressants in MG. Oral prednisolone is the most commonly used first-line drug. Corticosteroids are usually used as an interim measure while titrating up the doses of other immunosuppressants and waiting for those immunosuppressants to take full effect. A temporary worsening of MG known as a 'steroid dip' may occur if corticosteroids are started at a high dose. This steroid dip usually occurs 4 to 10 days after starting treatment and may precipitate a MG crisis. To overcome this problem, treatment should be started at a low dose on alternate days ${ }^{19}$ and gradually titrated upwards. ${ }^{20}$ In critically ill patients, high-dose daily corticosteroids can be started and additional short-term treatments such as intravenous immunoglobulin or plasma exchange can be used to overcome any temporary worsening. ${ }^{21}$ The potential adverse events from prolonged corticosteroid use necessitates a slow dose reduction to the minimum effective dose given on alternate days, ${ }^{20}$ but there is no clear evidence on the best time to reduce corticosteroids, how quickly or safely to do so, or how long patients should be kept on corticosteroids. ${ }^{21}$

Long-term corticosteroid use is associated with many adverse events, including cushingoid features, hypertension, 
diabetes, osteoporosis, infections, psychiatric disorders, insomnia, and elevations in white blood cell count. Calcium, vitamin D and bisphosphonate supplement should be started concurrently with corticosteroids to protect against osteoporosis. In some patients, corticosteroid-induced myopathy needs to be differentiated from myasthenic weakness.

\section{Long-term immunosuppression Azathioprine}

Azathioprine acts through its metabolite 6-mercaptopurine as a purine antagonist by inhibiting DNA synthesis and cell proliferation. There are several observational studies of azathioprine in MG. In a series of patients previously unresponsive to ACTH or glucocorticoids, $78 \%$ of 26 patients improved on azathioprine. ${ }^{22}$ In another study, $91 \%$ of 78 patients improved when treated with azathioprine, given alone or in combination with corticosteroids, thymectomy or both. ${ }^{23}$ Another study showed $83 \%$ of 18 patients improved with azathioprine treatment alone for more than 6 months. ${ }^{24}$ In a further study, all $41 \mathrm{MG}$ patients followed for more than 3 years improved when azathioprine was used either alone or in combination with prednisolone. ${ }^{25}$ In another study, $75 \%$ of 32 patients treated with azathioprine monotherapy improved compared with $70 \%$ of 57 patients treated with a combination of azathioprine and corticosteroids. ${ }^{26}$ The use of early 'high-dose' immunosuppression with azathioprine and prednisolone resulted in $50 \%$ of $\mathrm{MG}$ patients achieving remission after 2 years, compared with a remission rate of only $16 \%$ in those on a 'low-dose' regimen. ${ }^{27}$

A randomized unblinded trial of azathioprine plus initial prednisolone versus prednisolone alone in 41 patients with generalized MG showed less clinical deterioration within the first 60 months (the primary endpoint) in the azathioprine group compared to the prednisolone monotherapy group, although no differences in muscle strength measurements were seen between the two groups. ${ }^{28} \mathrm{~A}$ further randomized double-blind trial of azathioprine plus prednisolone versus prednisolone plus placebo in 34 patients with generalized MG reported no significant differences between the two treatment groups with regard to objective or subjective muscle strength measurements. ${ }^{29}$ In the same study, the median prednisolone dose did not differ significantly between the two treatment groups at 12 months, but was significantly reduced at 36 months in the azathioprine plus prednisolone group compared with the prednisolone plus placebo group, suggesting that azathioprine had a corticosteroid-sparing effect. ${ }^{29}$

In many countries, azathioprine is the first choice longterm immunosuppressant drug which is started together with corticosteroids to allow tapering of the latter to the lowest possible dose. Common adverse events of azathioprine are hepatotoxicity, nausea, vomiting, rash, cytopenia, and pancreatitis. Malignancy, primarily lymphoma, is a potential long-term complication but the absolute risk is difficult to ascertain because it is difficult to differentiate the effects of the drug from age-related increases in the background incidence of cancer. Patients with deficiency of thiopurine S-methyltransferase may develop severe bone marrowrelated toxicity. Red cell thiopurine S-methyltransferase activity can be measured to identify at risk patients prior to starting treatment.

\section{Cyclosporine}

The effect of cyclosporine is mediated by calcineurin inhibition of T-cell interleukin-2 production. There are three uncontrolled trials of cyclosporine in patients with severe MG. In one trial, where patients recruited were unresponsive either to acethylcholinesterase inhibitors alone or to the combination of thymectomy plus corticosteroids or azathioprine, $80 \%$ of ten patients showed marked improvement after 12 months of treatment. ${ }^{30}$ In another trial of patients who had failed to respond to thymectomy, corticosteroids, azathioprine, or all three, $78 \%$ of nine patients showed marked improvement after a mean of 2 years' treatment. ${ }^{31}$ In a third trial of patients who had not responded to thymectomy, corticosteroids and azathioprine, $85 \%$ of 52 patients showed marked improvement after an average follow-up duration of 30 months' treatment. $^{32}$

A randomized double-blind trial of cyclosporine monotherapy versus placebo in 20 patients with generalized $\mathrm{MG}$ reported significantly greater increases in muscle strength in the cyclosporine group compared with the placebo group both at 6 and 12 months. ${ }^{33}$ Another randomized double-blind trial of cyclosporine plus prednisolone versus prednisolone plus placebo in 39 patients with generalized MG demonstrated that at 6 months, the cyclosporine group had a significantly greater increase in muscle strength than the placebo group. ${ }^{34}$ Rather surprisingly, there was no significant difference in the percentage change of corticosteroid dose between the two groups at the end of 6 months; ${ }^{34}$ if cyclosporine is effective, it might be expected to have a corticosteroid-sparing effect.

Although cyclosporine has been shown to be clinically effective in MG, its use is limited by the potentially serious adverse events associated with this drug such as nephrotoxicity, hypertension, and malignancy. Other common adverse events include flu-like symptoms, gingival hyperplasia, hypertrichosis, myalgia, and tremor. 


\section{Cyclophosphamide}

Cyclophosphamide is a DNA-alkylating drug and nonspecific cell-cycle inhibitor. One study reported stable remissions in 42 patients who were given cyclophosphamide for 2 to 37 months. Of these patients, 33 received concomitant corticosteroid treatment and five underwent thymectomy. ${ }^{35}$ A randomized double-blind trial of intravenous pulsed cyclophosphamide plus prednisolone versus prednisolone plus placebo in 23 patients with severe generalized MG, demonstrated that cyclophosphamide significantly improved muscle strength at 12 months, but not at 6 months. The cyclophosphamide group also had significantly lower corticosteroid doses at 6 and 12 months than at the start of the trial. ${ }^{36}$

Although there is evidence of clinical efficacy of cyclophosphamide in MG, the use of the drug is restricted by its relatively high risk of severe adverse events such as bladder toxicity, bone marrow suppression, opportunistic infections, infertility, and malignancy. Other common adverse events associated with cyclophosphamide include nausea, vomiting, alopecia, abdominal pain, and diarrhea.

\section{Methotrexate}

Methotrexate is a folate antagonist that inhibits de novo synthesis of purines and pyrimidines. There are no good quality published studies of methotrexate in MG. However, methotrexate is still often used as second-line treatment in patients who do not tolerate or are unresponsive to azathioprine for several reasons: (a) data extrapolated from other autoimmune disorders suggest that it should be effective in $M G,{ }^{20}$ (b) expert panel guidelines support its use as second-line treatment in $\mathrm{MG},{ }^{20}$ and (c) many physicians have extensive personal experience of successfully using it in $\mathrm{MG} .{ }^{21} \mathrm{~A}$ clinical trial to assess the efficacy of methotrexate in $\mathrm{MG}$ is underway.

Adverse events associated with methotrexate are usually mild such as alopecia, mucositis, gastrointestinal intolerance, and mild elevation of liver enzymes. Nevertheless, severe adverse events including hepatotoxicity, hematopoietic suppression, and pneumonitis may sometimes occur.

\section{Mycophenolate mofetil}

Mycophenolate mofetil, when converted to its active metabolite mycophenolic acid, inhibits T-cell proliferation by blocking purine synthesis. Several open-label trials of mycophenolate mofetil in MG have been published. In one trial, $68 \%$ of 22 patients who were either previously unresponsive to azathioprine, on combination therapy with corticosteroids or on mycophenolate mofetil monotherapy, treated for between 2 and 18 months demonstrated marked improvement. ${ }^{37}$ In another trial, $67 \%$ of twelve patients who had not responded to corticosteroids, azathioprine, cyclosporine, or thymectomy showed marked improvement after 6 months of treatment. ${ }^{38}$ In a further trial, $59 \%$ of 32 patients who had been unresponsive to corticosteroids, azathioprine, cyclosporine, methotrexate, or thymectomy showed marked improvement after an average of 11 months' treatment. ${ }^{39}$ A retrospective analysis of mycophenolate mofetil in $85 \mathrm{MG}$ patients, 48 of whom had undergone thymectomy and 66 of whom were on various combinations of corticosteroids, azathioprine, cyclosporine, and methotrexate, showed an improvement in $73 \%$ of patients. ${ }^{40}$ Another retrospective study of MG patients examined the effect of mycophenolate mofetil either as monotherapy or in combination with corticosteroids. In the monotherapy group, approximately $75 \%$ of 36 patients achieved a desirable outcome after 25 months of treatment, compared with approximately $16 \%$ when mycophenolate mofetil was initially started. In the mycophenolate mofetil plus corticosteroids group, approximately $75 \%$ of 66 patients achieved a desirable outcome after 24 months of treatment, compared with approximately $21 \%$ when mycophenolate mofetil was initially started. ${ }^{41}$

A randomized, double-blind trial of mycophenolate mofetil plus corticosteroids versus corticosteroids plus placebo of $176 \mathrm{MG}$ patients for 36 weeks did not show efficacy for mycophenolate mofetil. ${ }^{42}$ Similarly, a second randomized double-blind trial of mycophenolate mofetil plus corticosteroids versus corticosteroids plus placebo of $80 \mathrm{MG}$ patients for 12 weeks failed to demonstrate efficacy for mycophenolate mofetil. ${ }^{43}$

The results of the two randomized controlled trials of mycophenolate mofetil were considered limited by their relatively short duration and greater than predicted benefit from the prednisolone doses used in the placebo arms of the studies. ${ }^{44,45}$ Since the drug is normally well tolerated with a relatively good adverse effect profile, it can be considered as third-line treatment in MG. The adverse events of mycophenolate mofetil are usually mild such as headache, nausea, and diarrhea, but more serious adverse events including infections, hematopoietic suppression, and hepatotoxicity can occasionally occur. Progressive multifocal leukoencephalopathy has been associated with mycophenolate mofetil treatment.

\section{Rituximab}

Rituximab is a chimeric monoclonal antibody against the B-cell surface marker CD20. In one series, six patients with 
refractory MG (four MuSK antibody positive, two AChR antibody positive) were treated with rituximab, resulting in a decreased need for immunosuppressant treatment, improved clinical function or both in all patients. ${ }^{46}$ In another series, five patients with generalized MG resistant to conventional immunosuppression were treated with rituximab, with good clinical improvement reported in all. ${ }^{47}$ In a retrospective study of ten patients with generalized MG (three MuSK antibody positive, seven AChR antibody positive) refractory to conventional immunosuppressant treatment, rituximab resulted in $60 \%$ of patients (including all the MuSK antibody positive cases) improving clinically or being able to decrease immunosuppressant treatment. ${ }^{48}$ Another retrospective study of low-dose rituximab in refractory $\mathrm{MG}$ reported clinical improvement in $79 \%$ of the 14 patients (three MuSK antibody positive, eleven AChR antibody positive), including all MuSK antibody positive cases. ${ }^{49}$

There is preliminary evidence that rituximab is beneficial in $\mathrm{MG}$, but it should only be used in severe refractory cases unresponsive to other treatments. Ongoing trials are evaluating its use. The current prohibitive cost of this drug is a major disadvantage. Common infusion-related adverse events associated with rituximab are fever, chills, nausea, vomiting, flushing, and bronchospasm. Other more severe adverse events include neutropenia, infections, and the risk of progressive multifocal leukoencephalopathy.

\section{Tacrolimus}

Tacrolimus inhibits T-cell and interleukin-2 production via the calcineurin-mediated pathway. In a 16-week, openlabel trial of 19 patients with generalized MG treated with low-dose tacrolimus, all of whom had previously undergone thymectomy and all but one of whom were on corticosteroid treatment, $37 \%$ showed clinical improvement at the end of the study.$^{50}$ Furthermore, $67 \%$ of twelve patients from the previous study that continued tacrolimus for up to 2 years showed clinical improvement. ${ }^{51}$ In another open trial of low-dose tacrolimus in thymectomized and corticosteroiddependent patients, $71 \%$ of the 17 patients treated improved clinically. ${ }^{52}$ In a further open-label study of tacrolimus in severe $\mathrm{MG}$, where all patients were on prednisolone and cyclosporine, and had undergone thymectomy, $87 \%$ of the 79 patients in the trial achieved pharmacological remission after a mean follow-up of 2.5 years. ${ }^{53}$ Another study examined the use of low-dose tacrolimus in $212 \mathrm{MG}$ patients. These patients were either thymectomized, cyclosporine- and prednisolone-dependent patients, or thymectomized patients who started tacrolimus early postoperatively, or patients over 60 years old with nonthymomatous generalized MG or in whom thymectomy was contraindicated. This study, with a mean follow-up of 49.3 months, showed that muscle strength increased $23 \%$ after 1 month of treatment and $29 \%$ at the end of the study. ${ }^{54} \mathrm{~A}$ further published study of ten corticosteroid-dependent generalized MG patients treated with low-dose tacrolimus for a mean of 3.1 years reported that $50 \%$ of patients improved clinically at the end of the study. ${ }^{55}$ However, another study reported no significant clinical improvement in nine corticosteroid-dependent generalized $\mathrm{MG}$ patients treated with low-dose tacrolimus for 5 years. ${ }^{56}$ In another study, 86\% of 47 generalized MG patients on low-dose tacrolimus over 24 weeks showed clinical improvement. ${ }^{57}$

One randomized unblinded nonplacebo controlled trial of tacrolimus plus corticosteroids with or without plasma exchange versus no tacrolimus plus corticosteroids with or without plasma exchange in 34 generalized MG patients showed that tacrolimus reduced the need for other immunotherapy such as plasma exchange and corticosteroids. ${ }^{58}$

Tacrolimus appears to be relatively safe at doses used in $\mathrm{MG}$ and can be considered as third-line treatment. Common adverse events include mild elevation of serum creatinine, hypertension, headache, hyperglycemia, tremor, parasthesia, decreased lymphocyte counts, and raised neutrophil counts. Tacrolimus may increase the risk of malignancy.

\section{Rapid short-term immunomodulation Intravenous immunoglobulin}

The mode of action of intravenous immunoglobulin in $\mathrm{MG}$ is incompletely understood, but key mechanisms include interference of signalling via Fc receptors, neutralization of activated complement, suppression of idiotypic antibodies, and modulation of proinflammatory cytokines. Intravenous immunoglobulin was first used in the 1980s in MG. ${ }^{59,60}$ Two reviews which collated results from previously published uncontrolled studies showed that intravenous immunoglobulin improved MG in more than $70 \%$ of cases. ${ }^{61,62}$ Two open studies of a total of 21 patients with severe generalized MG showed improvement with intravenous immunoglobulin in all patients. ${ }^{63,64}$ Intravenous immunoglobulin has been shown to have comparable effectiveness to plasma exchange in preoperative thymectomy preparation of MG patients. ${ }^{65,66}$

Five randomized controlled trials comparing intravenous immunoglobulin with placebo or other treatments in $\mathrm{MG}$ have been carried out. Two trials compared intravenous immunoglobulin to placebo. One trial of 15 patients 
with mild-to-moderate generalized MG showed no significant difference between the two groups at 6 weeks. In the same study, the clinical efficacy of intravenous immunoglobulin was similar whether $1.2 \mathrm{~g} / \mathrm{kg}$ or $2 \mathrm{~g} / \mathrm{kg}$ of intravenous immunoglobulin was used. ${ }^{67}$ In the other trial of 51 patients with acute exacerbation of generalized MG, intravenous immunoglobulin significantly improved muscle strength only in the group of patients with severe disease. ${ }^{68}$ Two trials compared intravenous immunoglobulin to plasma exchange. In the first trial with 87 patients with acute exacerbation of generalized $\mathrm{MG}$, no significant change in muscle strength between day 0 and day 15 was observed between the two treatment groups. ${ }^{69}$ The second trial with a crossover design of 12 patients with moderate-to-evere MG in a stable phase showed no significant difference between the two groups after 1 and 4 weeks of treatment. ${ }^{70}$ The Cochrane group reported that an unpublished, randomized controlled trial of intravenous immunoglobulin versus oral methylprednisolone in 33 patients with acute exacerbation of generalized MG failed to demonstrate any significant difference between the two treatment arms. ${ }^{71}$ Another randomized controlled trial comparing two doses of intravenous immunoglobulin in 173 patients with acute exacerbation of generalized MG did not demonstrate any significant difference in efficacy between the doses of $1 \mathrm{~g} / \mathrm{kg}$ and $2 \mathrm{~g} / \mathrm{kg}^{72}$

There is some evidence that intravenous immunoglobulin is efficacious in acute severe exacerbation of generalized $\mathrm{MG}$, but the evidence is less clear in chronic cases. ${ }^{73}$ Intravenous immunoglobulin is typically used in acute exacerbation of MG or to optimize muscle strength before surgery. Common adverse events associated with intravenous immunoglobulin include headache, nausea, fever, and allergic reaction. In patients with IgA deficiency which may be present in 1 in 1000 people, a severe anaphylactic reaction might occur. Volume overload is a risk in cardiomyopathy and soluteinduced renal failure may occur in patients with pre-existing renal impairment. High infusion rates may be associated with thrombotic complications such as myocardial infarction and stroke. However, the rate of adverse events from intravenous immunoglobulin appears to be less severe than those from plasma exchange. ${ }^{69,70}$

\section{Plasma exchange}

Plasma exchange is thought to work in MG by removing circulating antibodies, cytokines, immune complexes, and other inflammatory mediators. In $\mathrm{MG}$, the concentration of AChR and MuSK antibodies has been shown to decrease with plasma exchange. ${ }^{74,75}$ Plasma exchange was first used in the $1970 \mathrm{~s}$ in $\mathrm{MG} .{ }^{76}$ Several relatively large open studies of 20 or more patients, most of whom were already on other immunosuppressive or immunomodulatory treatment, have shown benefit from plasma exchange. Of 166 patients with generalized $\mathrm{MG}$ from five studies, 78\% reported improvement with plasma exchange. ${ }^{77-81}$ Three fairly large retrospective studies totaling 84 patients with generalized MG showed improvement in $96 \%$ of patients. ${ }^{82-84}$ Another retrospective study of plasma exchange versus intravenous immunoglobulin in myasthenic crisis, demonstrated that the ventilatory status at 2 weeks and functional outcome after 1 month was better in the plasma exchange group. ${ }^{85}$ A nonrandomized trial comparing different plasma exchange protocols in generalized $\mathrm{MG}$ failed to show any significant difference in efficacy between the treatments. ${ }^{86}$ Prethymectomy plasmapheresis improves outcome after thymectomy in $\mathrm{MG}^{87,88}$

One randomized controlled trial of plasma exchange versus prednisolone monotherapy in 14 patients with generalized MG did not show any significant difference in muscle strength between the two treatment arms after 1 month. ${ }^{89}$ Two further randomized controlled trials comparing daily and alternate day plasma exchange in generalized MG did not show any significant difference in efficacy between the treatments..$^{90,91}$

Plasma exchange is commonly used in acute severe exacerbation of MG to achieve temporary improvement or as a method of optimizing MG control before surgery. The choice between plasma exchange and intravenous immunoglobulin is often based on the physician's opinion of the ability of a patient to tolerate each treatment. Since intravenous immunoglobulin is easier to administer, and associated with fewer adverse events than plasma exchange, and the efficacy of the two treatments is similar, the former is usually preferred to the latter. ${ }^{73}$ Most adverse events associated with plasma exchange are due to problems with vascular access such as infection, thrombosis, pneumothorax, and air embolism. Excessive fluid volume shift can result in hypotension or fluid overload and congestive cardiac failure. Citrate infused for anticoagulation may cause disturbances in acid-base homeostasis and hypocalcemia.

\section{Long-term immunomodulation Thymectomy}

The mechanism by which thymectomy potentially benefits non-thymomatous patients with generalized $\mathrm{MG}$ is incompletely understood, but may involve disrupting B-cells 
producing AChR antibodies. Although thymectomy has been the mainstay of treatment for non-thymomatous MG since the middle of the last centuary, a rigorous, evidence-based evaluation concluded that it only 'might' improve the chance of remission. ${ }^{92}$ An ongoing study is examining whether the addition of thymectomy to prednisolone monotherapy in AChR antibody positive patients leads to an overall reduction in the dose of prednisolone over 3 years.

Most retrospective studies indicate a better response to thymectomy when it is performed early in the disease course of generalized MG; thus the procedure is usually recommended within the first 3 years of diagnosis. ${ }^{93,94}$ There is no consensus on the age of non-thymomatous patients who should undergo thymectomy, but most experts restrict the procedure to patients under the age of 60-65 years because older patients usually have an atrophic thymus. ${ }^{93,94}$ Numerous approaches to thymus removal have been advocated, but the procedure that allows the greatest removal of thymic tissue would be expected to be the preferred option. Thymectomy is not recommended in MuSK antibody positive patients because retrospective analyses indicate a lack of typical thymus pathology in these patients. ${ }^{95}$ Whether a small proportion of seronegative MG patients do occasionally benefit from thymectomy remains controversial.

Thymectomy is always indicated in patients with thymoma to resect the neoplasm and treat the MG which occurs in about $10 \%$ of cases. Tumor removal in these cases does not always lead to remission and, indeed, the disease course in thymomatous patients is commonly more severe than in nonthymomatous patients. Local irradiation and chemotherapy may be needed in certain more aggressive thymoma cases. Long-term monitoring for recurrence with computed tomography or magnetic resonance imaging may be needed.

\section{Emerging treatments}

A number of potentially useful treatments show promise in animal models ${ }^{96}$ and theoretically. ${ }^{97}$

Animal models, such as the experimental autoimmune MG (EAMG) model which can be induced in different species, have been used to investigate several therapeutic possibilities. The most widely used models nowadays are rodents, and active immunization is with AChR from Torpedo electric organ which is a rich source of the receptor. In rats, both the acute and chronic phases of MG are present, as in humans, although the thymus is not involved. ${ }^{98}$ EAMG in mice is harder to induce, but mouse models have the advantage of the availability of mouse-specific reagents and knock-out strains which allow analyses which cannot be carried out in rats.
Different derivatives of AChR have been tested as potential therapeutic agents in EAMG. The first successful antigenspecific immunomodulation of EAMG was performed in 1978 with an intradermal injection of a chemically modified Torpedo AChR, where the denatured AChR derivative both prevented the induction of EAMG and suppressed the ongoing disease in rabbits. ${ }^{99}$ Native Torpedo AChR has also been shown to modulate EAMG, ${ }^{100-102}$ but the highly immunogenic nature of this native $\mathrm{AChR}^{103,104}$ has hampered its therapeutic application.

Advances in cloning and genetic engineering have led to the discovery that a certain region of the AChR molecule, termed the main immunogenic region, is the target of a large portion of the antibodies to AChR. ${ }^{105}$ Recombinant allogenic or syngeneic fragments to the appropriate regions of AChR have been shown to suppress acute and chronic manifestations of rat EAMG. ${ }^{106-109}$ It is thought that this approach suppresses autoimmune MG by inducing tolerance toward the self-autoantigen, rather than the directly neutralizing antibodies reacting with the autoantigen.

The use of synthetic peptides against immunodominant $\mathrm{T}$ - and B-cell epitopes within the AChR molecule is another potentially useful approach in MG. Several studies using such peptides have been associated with the amelioration of or protection from EAMG in rodent models. ${ }^{110-113}$ The autoimmune response in MG is polyclonal, thus peptides which represent only one or two dominant epitopes are unlikely to be effective. However, the use of peptide mixtures has been shown to be ineffective in rats with EAMG, possibly due to their inability to meet conformation requirements even when they are relatively long. ${ }^{114}$

Several approaches to influence key players in the immune process such as regulatory T-cells, ${ }^{115}$ dendritic cells, ${ }^{116}$ cytokines, ${ }^{117}$ costimulatory molecules, ${ }^{118}$ and complement pathways ${ }^{119}$ have also been shown to ameliorate or prevent EAMG.

Several techniques have been devised as alternatives to plasma exchange. These include immunoadsorption of antiAChR antibodies using gels or columns. ${ }^{120-122}$ However, the nonspecificity of these approaches leads to the indiscriminate removal of all, or most, immunoglobulins, including potentially useful antibodies. More antigen-specific immunoadsorbent approaches have being explored, ${ }^{123-125}$ with mixed results.

\section{Conclusion}

There remain major gaps in our knowledge of the treatments used in generalized MG. Much of the evidence that 
we use in determining the choice of treatment derives from clinical experience, observational studies, and expert opinion. Treatment regimens vary among different physicians, even from the same country, because many decisions are made primarily on the experience and familiarity of the treating physician to a particular regime. Furthermore, organizing randomized controlled trials in MG is fraught with difficulties, not least that the rarity of the condition makes recruitment into trials a major obstacle. In addition, the phenotypical variability between $\mathrm{AChR}$ antibody positive, MuSK antibody positive and seronegative patients, and the fluctuating nature of $\mathrm{MG}$ are confounding factors in evaluating response to treatments. There is also a need to comparatively evaluate the effectiveness of current and emerging treatments in $\mathrm{MG}$.

Various studies in experimental models of $\mathrm{MG}$ aim to develop novel and improved treatments that would have less adverse events than currently used treatments. Many promising ways to modulate the immune system in EAMG have been examined, but not all of these would necessarily be successful in human patients. It is worth noting that a multifaceted approach to the management of MG, with both antigen-specific immunotherapy and the direct modulation of key immunological factors involved in the autoimmune process, is likely to yield better treatments to improve the management of MG.

\section{Disclosure}

The author declares no conflicts of interests in this work.

\section{References}

1. Grob D, Brunner N, Namba T, Pagala M. Lifetime course of myasthenia gravis. Muscle Nerve. 2008;37(2):141-149.

2. Phillips LH 2nd, Torner JC. Epidemiologic evidence for a changing natural history of myasthenia gravis. Neurology. 1996;47(5): 1233-1238.

3. Vincent A, Palace J, Hilton-Jones D. Myasthenia gravis. Lancet. 2001; 357(9274):2122-2128.

4. Grob D, Arsura EL, Brunner NG, Namba T. The course of myasthenia gravis and therapies affecting outcome. Ann N Y Acad Sci. 1987;505: 472-499.

5. Schwarz H. Mestinon (pyridostigmine bromide) in myasthenia gravis. Can Med Assoc J. 1956;75(2):98-100.

6. Simpson JF, Westerberg MR, Magee KR. Myasthenia gravis. An analysis of 295 cases. Acta Neurol Scand. 1966;42(Suppl 23):1-27.

7. Mehndiratta MM, Pandey S, Kuntzer T. Acetylcholinesterase inhibitor treatment for myasthenia gravis. Cochrane Database Syst Rev. 2011; (2):CD006986.

8. Hatanaka YH. Nonresponsiveness to anticholinesterase agents in patients with MuSK-antibody-positive MG. Neurology. 2005;65(9): 1508-1509.

9. Simon HE. Myasthenia gravis: effect of treatment with anterior pituitary extract. JAMA. 1935;104(23):2065-2066.

10. Genkins G, Kornfeld P, Osserman KE, Namba T, Grob D, Brunner NG. The use of ACTH and corticosteroids in myasthenia gravis. Ann NY Acad Sci. 1971;183:369-374.
11. Pascuzzi RM, Coslett HB, Johns TR. Long-term corticosteroid treatment of myasthenia gravis: report of 116 patients. Ann Neurol. 1984;15(3): 291-298.

12. Sghirlanzoni A, Peluchetti D, Mantegazza R, Fiacchino F, Cornelio F. Myasthenia gravis: prolonged treatment with steroids. Neurology. 1984; 34(2):170-174.

13. Cosi V, Citterio A, Lombardi M, Piccolo G, Romani A, Erbetta A. Effectiveness of steroid treatment in myasthenia gravis: a retrospective study. Acta Neurol Scand. 1991;84(1):33-39.

14. Evoli A, Batocchi AP, Palmisani MT, Lo Monaco M, Tonali P. Long-term results of corticosteroid therapy in patients with myasthenia gravis. Eur Neurol. 1992;32(1):37-43.

15. Bu B, Yang M, Xu J, Gao BT, Pan DJ, Cai YX. A prospective study of effectiveness and safety of long-term prednisone therapy in patients with myasthenia gravis. Zhonghua Shen Jing Ge Za Zhi. 2000;33: 28-31. Chinese.

16. Howard FM Jr, Duane DD, Lambert EH, Daube JR. Alternate-day prednisone: preliminary report of a double-blind controlled study. Ann NY Acad Sci. 1976;274:596-607.

17. Lindberg C, Andersen O, Lefvert AK. Treatment of myasthenia gravis with methylprednisolone pulse: a double blind study. Acta Neurol Scand. 1998;97(6):370-373.

18. Zhang J, Wu H. Effectiveness of steroid treatment in juvenile myasthenia gravis. Chinese Journal of Pediatrics. 1998;36(10):612-614.

19. Warmolts JR, Engel WK. Benefit from alternate-day prednisone in myasthenia gravis. $N$ Engl J Med. 1972;286(1):17-20.

20. Skeie GO, Apostolski S, Evoli A, et al. Guidelines for treatment of autoimmune neuromuscular transmission disorders. Eur J Neurol. 2010;17(7): 893-902.

21. Sathasivam S. Steroids and immunosuppressant drugs in myasthenia gravis. Nat Clin Pract Neurol. 2008;4(6):317-327.

22. Matell G, Bergstrom K, Franksson C, et al. Effects of some immunosuppressive procedures in myasthenia gravis. Ann NY Acad Sci. 1976;274: 659-676.

23. Mertens HG, Hertel G, Reuther P, Ricker K. Effect of immunosuppressive drugs (azathioprine). Ann NY Acad Sci. 1981;377:691-699.

24. Witte AS, Cornblath DR, Parry GJ, Lisak RP, Schatz NJ. Azathioprine in the treatment of myasthenia gravis. Ann Neurol. 1984;15(6): 602-605.

25. Kuks JBM, Djojoatmodjo S, Oosterhuis HJHG. Azathioprine myasthenia gravis: observations in 41 patients and a review of literature. Neuromuscul Disord. 1991;1(6):423-431.

26. Mantegazza R, Antozzi C, Peluchetti D, Sghirlanzoni A, Cornelio F. Azathioprine as a single drug or in combination with steroids in the treatment of myasthenia gravis. J Neurol. 1988;235(8):449-453.

27. Heckmann JM, LeePan EB, Eastman RW. High-dose immunosuppressive therapy in generalized myasthenia gravis - a 2-year follow-up study. S Afr Med J. 2001;91(9):765-770.

28. Myasthenia Gravis Clinical Study Group. A randomised clinical trial comparing prednisolone and azathioprine in myasthenia gravis. Results of a second interim analysis. J Neurol Neurosurg Psychiatry. 1993; 56(11):1157-1163.

29. Palace J, Newsom-Davis J, Lecky B. A randomized double-blind trial of prednisolone alone or with azathioprine in myasthenia gravis. Neurology. 1998;50(6):1778-1783.

30. Goulon M, Elkharrat D, Lokiec F, Gajdos P. Results of a one-year open trial of cyclosporine in ten patients with severe myasthenia gravis. Transplant Proc. 1988;20(3 Suppl 4):211-217.

31. Bonifati DM, Angelini C. Long-term cyclosporine treatment in a group of severe myasthenia gravis patients. J Neurol. 1997;244(9):542-547.

32. Lavrnic D, Vujic A, Rakocevic-Stojanovic V, et al. Cyclosporine in the treatment of myasthenia gravis. Acta Neurol Scand. 2005;111(4): 247-252.

33. Tindall RSA, Rollins JA, Phillips JT, Greenlee RG, Wells L, Belendiuk G. Preliminary results of a double-blind, randomized, placebo-controlled trial of cyclosporine in myasthenia gravis. $N$ Engl J Med. 1987;316(12):719-724. 
34. Tindall RS, Phillips JT, Rollins JA, Wells L, Hall K. A clinical therapeutic trial of cyclosporine in myasthenia gravis. Ann NY Acad Sci. 1993;681:539-551.

35. Perez MC, Buot WL, Mercado-Danguilan C, Bababaldo ZG, Renales LD. Stable remissions in myasthenia gravis. Neurology. 1981; 31(1):32-37.

36. De Feo LG, Schottlender J, Martelli NA, Molfino NA. Use of intravenous pulsed cyclophosphamide in severe, generalized myasthenia gravis. Muscle Nerve. 2002;26(1):31-36.

37. Cos L, Mankodi AK, Tawil R, Thornton CA. Mycophenolate mofetil (MyM) is safe and well tolerated in myasthenia gravis (MG). Neurology. 2000;54(Suppl 3):A137.

38. Ciafaloni E, Massey JM, Tucker-Lipscomb B, Sanders DB. Mycophenolate mofetil for myasthenia gravis: an open-label pilot study. Neurology. 2001;56(1):97-99.

39. Chaudhry V, Cornblath DR, Griffin JW, O'Brien R, Drachman DB. Mycophenolate mofetil: a safe and promising immunosuppressant in neuromuscular diseases. Neurology. 2001;56(1):94-96.

40. Meriggioli MN, Ciafaloni E, Al-Hayk KA, et al. Mycophenolate mofetil for myasthenia gravis: an analysis of efficacy, safety, and tolerability. Neurology. 2003;61(10):1438-1440.

41. Hehir MK, Burns TM, Alpers J, Conaway MR, Sawa M, Sanders DB. Mycophenolate mofetil in AChR-antibody-positive myasthenia gravis: outcomes in 102 patients. Muscle Nerve. 2010;41(5):593-598.

42. Sanders DB, Hart IK, Mantegazza R, et al. An international, phase III, randomized trial of mycophenolate mofetil in myasthenia gravis. Neurology. 2008;71(6):400-406.

43. Muscle Study Group. A trial of mycophenolate mofetil with prednisone as initial immunotherapy in myasthenia gravis. Neurology. 2008;71(6): 394-399.

44. Sanders DB, Siddiqi ZA. Lessons from two trials of mycophenolate mofetil in myasthenia gravis. Ann NY Acad Sci. 2008;1132:249-253.

45. Phan C, Sanders DB, Siddiqi ZA. Mycophenolate mofetil in myasthenia gravis: the unanswered question. Expert Opin Pharmacother. 2008; 9(14):2545-2551.

46. Zebardast N, Patwa HS, Novella SP, Goldstein JM. Rituximab in the management of refractory myasthenia gravis. Muscle Nerve. 2010; 41(3):375-378.

47. Lindberg C, Bokawera M. Rituximab for severe myasthenia gravis - experience from five patients. Acta Neurol Scand. 2010;122(4): 225-228.

48. Maddison P, McConville J, Farrugia ME, et al. The use of rituximab in myasthenia gravis and Lambert-Eaton myasthenic syndrome. J Neurol Neurosurg Psychiatry. 2011. In press.

49. Blum S, Gillis D, Brown H, et al. Use and monitoring of low dose rituximab in myasthenia gravis. J Neurol Neurosurg Psychiatry. 2011;82(6): 659-663.

50. Konishi T, Yoshiyama Y, Takamori M, et al. Clinical study of FK506 in patients with myasthenia gravis. Muscle Nerve. 2003;28(5): 570-574.

51. Konishi T, Yoshiyama Y, Takamori M, Saida T, and the Japanese FK506 MG Study Group. Long-term treatment of generalised myasthenia gravis with FK506 (tacrolimus). J Neurol Neurosurg Psychiatry. 2005; $76(3): 448-450$.

52. Kawaguchi N, Yoshiyama Y, Nemoto Y, Munakata S, Fukutake T, Hattori T. Low-dose tacrolimus treatment in thymectomised and steroid-dependent myasthenia gravis. Curr Med Res Opin. 2004;20(8): 1269-1273.

53. Ponseti JM, Azem J, Fort JM, et al. Long-term results of tacrolimus in cyclosporine- and prednisolone-dependant myasthenia gravis. Neurology. 2005;64(9):1641-1643.

54. Ponseti JM, Gamez J, Azem J, Lopez-Cano M, Vilallonga R, Armengol M. Tacrolimus for myasthenia gravis. A clinical study of 212 patients. Ann NY Acad Sci. 2008;1132:254-263.

55. Nagaishi A, Yukitake M, Kuroda Y. Long-term treatment of steroiddependent myasthenia gravis patients with low-dose tacrolimus. Intern Med. 2008;47(8):731-736.
56. Minami N, Fukiji N, Doi S, et al. Five-year follow-up with low-dose tacrolimus in patients with myasthenia gravis. J Neurol Sci. 2011; 300(1-2):59-62.

57. Zhao CB. Zhang X, Zhang H, et al. Clinical efficacy and immunological impact of tacrolimus in Chinese patients with generalized myasthenia gravis. Int Immunopharmacol. 2011;11(4):519-524.

58. Nagane Y, Utsugisawa K, Obara D, Kondoh R, Terayama Y. Efficacy of low-dose FK506 in the treatment of myasthenia gravis - a randomized pilot study. Eur Neurol. 2005;53(3):146-150.

59. Fateh-Moghadam A, Wick M, Besinger U, Geursen RG. High dose intravenous gammaglobulin for myasthenia gravis. Lancet. 1984; 1(8381):848-849

60. Gajdos P, Outin HD, Elkharrat D, et al. High dose intravenous gammaglobulin for myasthenia gravis. Lancet. 1984;1(8373): 406-407.

61. Arsura EL. Experience with intravenous immunoglobulin in myasthenia gravis. Clin Immunol Immunopathol. 1989;53(2 Pt 2):S170-S179.

62. van der Merche FG, van Doorn PA. The current place of high-dose immunoglobulins in the treatment of neuromuscular disorders. Muscle Nerve. 1997;20(2):136-147.

63. Achiron A, Barak Y, Miron S, Sarova-Pinhas I. Immunoglobulin treatment in refractory myasthenia gravis. Muscle Nerve. 2000;23(4): $551-555$.

64. Hilkevich O, Drory VE, Chapman J, Korczyn AD. The use of intravenous immunoglobulin as maintenance therapy in myasthenia gravis. Clin Neuropharmacol. 2001;24(3):173-176.

65. Perez-Nellar J, Dominquez AM, Llorens-Figueroa JA, et al. A comparative study of intravenous immunoglobulin and plasmapheresis preoperatively in myasthenia. Rev Neurol. 2001;33(5):413-416.

66. Jensen P, Bril V. A comparison of the effectiveness of intravenous immunoglobulin and plasma exchange as preoperative therapy of myasthenia gravis. J Clin Neuromuscul Dis. 2008;9(3):352-355.

67. Wolfe GI, Barohn RJ, Foster BM, et al. Myasthenia Gravis-IVIG Study Group. Randomized, controlled trial of intravenous immunoglobulin in myasthenia gravis. Muscle Nerve. 2002;26(4):549-552.

68. Zinman L, Ng E, Bril V. IV immunoglobulin in patients with myasthenia gravis. A randomized controlled trial. Neurology. 2007;68(11): 837-841.

69. Gajdos P, Chevret S, Clair B, Tranchant C, Chastang C. Clinical trial of plasma exchange and high dose immunoglobulin in myasthenia gravis. Ann Neurol. 1997;41(6):789-796.

70. Ronager J, Ravnborg M, Hermansen I, Volstrup S. Immunoglobulin treatment versus plasma exchange in patients with chronic moderate to severe myasthenia gravis. Artif Organs. 2001;25(12):967-973.

71. Gajdos $P$, Chevret $S$, Toyka K. Intravenous immunoglobulin for myasthenia gravis. Cochrane Database Syst Rev. 2008;(1):CD002277.

72. Gajdos P, Tranchant C, Clair B, et al. Treatment of myasthenia gravis exacerbation with intravenous immunoglobulin $1 \mathrm{~g} / \mathrm{kg}$ versus $2 \mathrm{~g} / \mathrm{kg}$ : a randomized double blind clinical trial. Arch Neurol. 2005;62(11): 1689-1693.

73. Sathasivam S. Evidence for use of intravenous immunoglobulin and plasma exchange in generalised mysasthenia gravis. Adv Clin Neurosci Rehabil. 2009;9(1):8-10.

74. Thorlacius S, Lefvert AK, Aarli JA, et al. Plasma exchange in myasthenia gravis: effect on anti-AChR antibodies and other autoantibodies. Acta Neurol Scand. 1986;74(6):486-490.

75. Yeh JH, Chen WH, Chiu HC, Bai CH. MuSK antibody clearance during serial sessions of plasmapheresis for myasthenia gravis. J Neurol. 2007; 263(1-2):191-193.

76. Pinching AS, Peters DK. Remission of myasthenia gravis following plasma exchange. Lancet. 1976;2(8000):1373-1376.

77. Behan PO, Shakir RA, Simpson JA, Burnett AK, Allan TL, Haase AG. Plasma exchange combined with immunosuppressive therapy in myasthenia gravis. Lancet. 1979;2(8140):438-440.

78. Dau C. Response to plasmapheresis and immunosuppressive drug therapy in sixty myasthenia gravis patients. Ann NY Acad Sci. 1981;377: 700-708. 
79. Olarte MR, Shoenfeldt RS, Penn AS, Lovelace RE, Rowland LP. Effect of plasmapheresis in myasthenia gravis. Ann N Y Acad Sci. 1981;377: $725-728$.

80. Fornasari PM, Riva G, Piccolo G, Cosi V, Lombardi M. Short and long-term clinical effects of plasma exchange in 33 cases of myasthenia gravis. Int J Artif Organs. 1985;8(3):159-162.

81. Huo JT, Long HL, Luo X, Xu JZ. Clinical study of double filtration plasmapheresis for treatment of refractory myasthenia gravis. Nan Fang Yi Ke DA Xue Xue Bao [Journal of Southern Medical University]. 2007;27(3):355-357. Chinese.

82. Lazo-Langner A, Espinosa-Poblano I, Tirado-Cardenas N, et al. Therapeutic plasma exchange in Mexico: experience from a single institution. Am J Hematol. 2002;70(1):16-21.

83. Carandina-Maffeis R, Nucci A, Marques JF Jr, et al. Plasmapheresis in the treatment of myasthenia gravis: retrospective study of 26 patients. Arq Neuropsiquiatr. 2004;62(2B):391-395.

84. Yucesan C, Arslan O, Arat M, et al. Therapeutic plasma exchange in the treatment of neuroimmunologic disorders: review of 50 cases. Transfus Apher Sci. 2007;36(1):103-107.

85. Qureshi AI, Choudhry MA, Akbar MS, et al. Plasma exchange versus intravenous immunoglobulin treatment in myasthenic crisis. Neurology. 1999;52(3):629-632.

86. Mantegazza R, Bruzzone E, Regi B, et al. Single donor plasma in therapeutic exchange for myasthenia gravis. Int J Artif Organs. 1987; 10(5):315-318.

87. Nagayasu T, Yamayoshi T, Matsumoto K, et al. Beneficial effects of plasmapheresis before thymectomy on the outcome in myasthenia gravis. Jpn J Thorac Cardiovasc Surg. 2005;53(1):2-7.

88. Yeh JH, Chen WH, Huang KM, Chiu HC. Prethymectomy plasmapheresis in myasthenia gravis. J Clin Apher. 2005;20(4):217-221.

89. Gajdos P, Simon N, de Rohan-Chabot P, Raphael JC, Goulon M. Longterm effects of plasma exchange in myasthenia. Results of a randomized study. Presse Med. 1983;12(15):939-942.

90. Yeh JH, Chiu HC. Plasmapheresis in myasthenia gravis. A comparative study of daily versus alternately daily schedule. Acta Neurol Scand. 1999;99(3):147-151.

91. Trikha I, Singh S, Goyal V, Shukla G, Bhasin R, Behari M. Comparative efficacy of low dose daily versus alternate day plasma exchange in severe myasthenia gravis: a randomised trial. J Neurol. 2007; 254(8):989-995.

92. Gronseth GS. Barohn RJ. Practice parameter: thymectomy for autoimmune myasthenia gravis (an evidence-based review): report of the Quality Standards Subcommittee of the American Academy of Neurology. Neurology. 2000;55(1):7-15.

93. Gold R, Schneider-Gold R. Current and future standards in treatment of myasthenia gravis. Neurotherapeutics. 2008;5(4):535-541.

94. Kumar V, Kaminski HJ. Treatment of myasthenia gravis. Curr Neurol Neurosci Rep. 2011;11(1):89-96.

95. Leite MI, Strobel P, Jones M, et al. Fewer thymic changes in MuSK antibody positive than in MuSK antibody-negative MG. Ann Neurol. 2005;57(3):444-448.

96. Souroujon MC, Brenner T, Fuchs S. Development of novel therapies for MG: studies in animal models. Autoimmunity. 2010;43(5-6): $446-460$.

97. Lagoumintzis G, Zisimopoulou P, Kordas G, Lazaridis K, Poulas K, Tzartos SJ. Recent approaches to the development of antigen-specific immunotherapies for myasthenia gravis. Autoimmunity. 2010;43(5-6): 436-445.

98. Meinl E, Klinkert WE, Wekerle H. The thymus in myasthenia gravis. Changes typical for the human disease are absent in experimental autoimmune myasthenia gravis of the Lewis rat. Am J Pathol. 1991;139(5): 995-1008

99. Bartfeld D, Fuchs S. Specific immunosuppression of experimental autoimmune myasthenia gravis by denatured acetylcholine receptor. Proc Natl Acad Sci U S A. 1978;75(8):4006-4010.
100. Wang ZY, Qiao J, Link H. Suppression of experimental autoimmune myasthenia gravis by oral administration of acetylcholine receptor. J Neuroimmunol. 1993;44(2):209-214.

101. Okumura S, McIntosh K, Drachman DB. Oral administration of acetylcholine receptor: effects on experimental myasthenia gravis. Ann Neurol. 1994;36(5):704-713.

102. Ma CG, Zhang GX, Xiao BG, Link J, Olsson T, Link H. Suppression of experimental autoimmune myasthenia gravis by nasal administration of acetylcholine receptor. J Neuroimmunol. 1995;58(1):51-60.

103. Drachman DB, Okumura S, Adams RN, McIntosh KR. Oral tolerance in myasthenia gravis. Ann NY Acad Sci. 1996;778:258-272.

104. Shi FD, Bai XF, Li HL, Huang YM, Van der Meide PH, Link H. Nasal tolerance in experimental autoimmune myasthenia gravis (EAMG): induction of protective tolerance in primed animals. Clin Exp Immunol. 1998;111(3):506-512.

105. Tzartos SJ, Lindstrom JM. Monoclonal antibodies used to probe acetylcholine receptor structure: localization of the main immunogenic region and detection of similarities between subunits. Proc Natl Acad Sci US A. 1980;77(2):755-759.

106. Im SH, Barchan D, Fuchs S, Souroujan MC. Suppression of ongoing experimental myasthenia by oral treatment with an acetylcholine receptor recombinant fragment. J Clin Invest. 1999;104(12): 1723-1730.

107. Barchan D, Souroujon MC, Im SH, Antozzi C, Fuchs S. Antigen-specific modulation of experimental myasthenia gravis: nasal tolerization with recombinant fragments of the human acetylcholine receptor alpha-subunit. Proc Natl Acad Sci U S A. 1999;96(14):8086-8091.

108. Im SH, Barchan D, Fuchs S, Souroujon MC. Mechanism of nasal tolerance induced by a recombinant fragment of acetylcholine receptor for treatment of experimental myasthenia gravis. $J$ Neuroimmunol. 2000;111(1-2):161-168.

109. Maiti PK, Feferman T, Im SH, Souroujon MC, Fuchs S. Immunosuppression of rat myasthenia gravis by oral administration of a syngeneic acetylcholine receptor fragment. J Neuroimmunol. 2004;152(1-2): $112-120$.

110. Wu B, Deng C, Goluszko E, Christadoss P. Tolerance to a dominant $\mathrm{T}$ cell epitope in the acetylcholine receptor molecule induces epitope spread and suppresses murine myasthenia gravis. J Immunol. 1997; 159(6):3016-3023.

111. Karachunski PI, Ostlie NS, Okita DK, Garman R, Conti-Fine BM. Subcutaneous administration of T-epitope sequences of the acetylcholine receptor prevents experimental myasthenia gravis. J Neuroimmunol. 1999;93(1-2):108-121.

112. Shenoy M, Oshima M, Atassi MZ, Christadoss P. Suppression of experimental autoimmune myasthenia gravis by epitope-specific neonatal tolerance to synthetic region alpha 146-162 of acetylcholine receptor. Clin Immunol Immunopathol. 1993;66(3):230-238.

113. Baggi F, Andreetta F, Caspani E, et al. Oral administration of an immunodominant T-cell epitope downregulates Th1/Th2 cytokines and prevents experimental myasthenia gravis. J Clin Invest. 1999;104(9): 1287-1295.

114. Souroujon MC, Maiti PK, Feferman T, Im SH, Raveh L, Fuchs S. Suppression of myasthenia gravis by antigen-specific mucosal tolerance and modulation of cytokines and costimulatory factors. Ann NY Acad Sci. 2003;998:533-536.

115. Sheng JR, Li LC, Ganesh BB, Prabhakar BS, Meriggioli MN. Regulatory T cells induced by GM-CSF suppress ongoing experimental myasthenia gravis. Clin Immunol. 2008;128(2):172-180.

116. Yarilin D, Duan R, Huang YM, Xiao BG. Dendritic cells exposed in vitro to TGF-beta1 ameliorate experimental autoimmune myasthenia gravis. Clin Exp Immunol. 2002;127(2):214-219.

117. Im SH, Barchan D, Maiti PK, Raveh L, Souroujon MC, Fuchs S. Suppression of experimental myasthenia gravis, a B cell-mediated autoimmune disease, by blockade of IL-18. FASEB J. 2001;15(12): 2140-2148. 
118. Im SH, Barchan D, Maiti PK, Fuchs S, Souroujon MC. Blockade of CD40 ligand suppresses chronic experimental myasthenia gravis by down-regulation of Th1 differentiation and up-regulation of CTLA-4. J Immunol. 2001;166(11):6893-6898.

119. Soltys J, Kusner LL, Young A, et al. Novel complement inhibitor limits severity of experimentally myasthenia gravis. Ann Neurol. 2009;65(1): 67-75.

120. Yamazaki Z, Fujimori Y, Takahama T, et al. Efficiency and biocompatibility of a new immunosorbent. Trans Am Soc Artif Intern Organs. 1982;28:318-323.

121. Matic G, Winkler RE, Tiess M, Ramlow W. Selective apheresis-time for a change. Int J Artif Organs. 2001;24(1):4-7.

122. Ptak J. Changes of plasma proteins after immunoadsorption using Ig-Adsopak columns in patients with myasthenia gravis. Transfus Apher Sci. 2004;30(2):125-129.
123. Takamori M, Maruta T. Immunoadsorption in myasthenia gravis based on specific ligands mimicking the immunogenic sites of the acetylcholine receptor. Ther Apher. 2001;5(5):340-350.

124. Guo CY, Li ZY, Xu MQ, Yuan JM. Preparation of an immunoadsorbent coupled with a recombinant antigen to remove anti-acetylcholine receptor antibodies in abnormal serum. J Immunol Methods. 2005; 303(1-2):142-147.

125. Zisimopoulou P, Lagoumintzis G, Poulas K, Tzartos S. Antigen-specific apheresis of human anti-acetylcholine receptor autoantibodies from myasthenia gravis patients' sera using Escherichia coli-expressed receptor domains. J Neuroimmunol. 2008;200(1-2):133-141.

\section{Publish your work in this journal}

Therapeutics and Clinical Risk Management is an international, peerreviewed journal of clinical therapeutics and risk management, focusing on concise rapid reporting of clinical studies in all therapeutic areas, outcomes, safety, and programs for the effective, safe, and sustained use of medicines. This journal is indexed on PubMed Central, CAS,

\section{Dovepress}

EMBase, Scopus and the Elsevier Bibliographic databases. The manuscript management system is completely online and includes a very quick and fair peer-review system, which is all easy to use. Visit http://www.dovepress.com/testimonials.php to read real quotes from published authors.

Submit your manuscript here: http://www.dovepress.com/therapeutics-and-clinical-risk-management-journal 